\title{
Stability of gravity currents generated by finite-volume releases
}

\author{
By JOCHONIA S. MATHUNJWA AND ANDREW J. HOGG \\ Centre of Environmental and Geophysical Flows, School of Mathematics, University of Bristol, \\ University Walk, Bristol BS8 1TW, UK
}

(Received 28 June 2005 and in revised form 26 January 2006)

We generalize the linear stability analysis of the axisymmetric self-similar solution of gravity currents from finite-volume releases to include perturbations that depend on both radial and azimuthal coordinates. We show that the similarity solution is stable to sufficiently small perturbations by proving that all perturbation eigenfunctions decay in time. Moreover, asymmetric perturbations are shown to decay more rapidly than axisymmetric perturbations in general. An asymptotic formula for the eigenvalues is derived, which indicates that asymptotic rates of decay of perturbations are given by $t^{-\sigma}$ where $0<\sigma<\frac{1}{4}$ as the Froude number decreases from $\sqrt{2}$ to 0 . We demonstrate that this formula agrees closely with numerically calculated eigenvalues and, in the absence of azimuthal dependence, it reduces to an expression that improves on the asymptotic formula obtained by Grundy \& Rottman (1985). For two-dimensional (planar) currents, we further prove analytically that all perturbation eigenfunctions decay like $t^{-1 / 2}$.

\section{Introduction}

Inertial gravity currents occur in many natural and industrial situations, driven by density gradients which may be due to differences in fluid composition, phase, temperature or concentration of suspended particulate. For example, differences in salinity account for the spreading of fresh river water above seawater in estuarine environments, whilst the flow of sediment-laden turbidity currents along ocean floors is driven by differences in the concentration of particle suspensions. A comprehensive review of other examples is provided by Simpson (1997).

The first mathematical study of the motion of a high-Reynolds-number gravity current was carried out by von Kármán (1940). Using the Bernoulli equation, he established that the rate of advance of the front is a function of the height at the front of the gravity current and the specific ratio of the density difference between the two fluids. There have been numerous subsequent studies, both theoretical and experimental, to investigate flows produced by the instantaneous release of a finite volume of dense fluid within an ambient fluid of lower density (see, for example, Fay 1969; Fannelop \& Waldman 1972; Hoult 1972; Huppert \& Simpson 1980). In these studies, a model is developed which describes the flow of the current in the regime when the inertia associated with the moving fluid is balanced by the driving gravitational force arising from the density differences, while the force due to viscous drag is negligible. On the assumption that the thickness of the current is small compared with its length and with the depth of the ambient, this model employs the shallow-water approximation. The main assumptions of the shallow-water approach 
are that vertical accelerations are negligible and the pressure adopts a hydrostatic distribution. Moreover, the two fluids are assumed to be incompressible and miscible (so that surface-tension effects are unimportant) and any mixing between them is neglected. Laboratory experiments conducted by Hallworth et al. (1996) indicated that, while some entrainment (and hence mixing) occurs, the rate of advance of the front predicted by this model is in good agreement with experimental measurements, at least during the early stages of its motion.

A dynamic condition is required near the front because dissipation and vertical accelerations are no longer negligible and hence the shallow-water assumptions are not valid there. A Froude number condition, linking the frontal velocity with the height, is imposed (von Kármán 1940; Benjamin 1968; Huppert \& Simpson 1980). This condition has subsequently been utilized with success (see, for example, Hallworth, Hogg \& Huppert 1998; Hogg, Ungarish \& Huppert 2000; Shin, Dalziel \& Linden 2004).

When gravity currents are driven by compositional differences between the intruding fluid and the surrounding ambient they are termed homogeneous gravity currents and a self-similar solution of the shallow-water equations governing their evolution can be found (Fannelop \& Waldman 1972; Hoult 1972). The existence of this solution is based on the assumption that the density difference between the fluids remains constant as the flow develops and it indicates that currents with the same volume approach the same height profile in the intermediate asymptotic regime even if they evolve from different initial conditions. In the case of particle-driven currents however, particle sedimentation means that the density difference between the fluids is no longer constant in time, but declines progressively and the motion is not selfsimilar. Significant insight into the dynamics of these flows can be gained by deriving asymptotic series about the self-similar solution to obtain the deviations from the self-similar flow which describe the particle-driven currents (see, for example, Hogg et al. 2000; Harris, Hogg \& Huppert 2001). Although the regime in which these expansions are valid is usually limited, the first-order asymptotic functions derived using this approach provide valuable information about the structure of the solutions of the particle-driven currents and the way in which their evolution differs from the homogeneous currents of the same initial excess density.

Grundy \& Rottman (1985, referred to as GR herein) have studied the development of the self-similar solutions subsequent to the introduction of a small perturbation (disturbance), for both planar and axisymmetric gravity currents, and established that they are linearly stable. By imposing a disturbance with two-dimensional and radial symmetry in the planar and axisymmetric cases, respectively, they showed that all eigenfunctions of the disturbance decay in time. They also derived asymptotic formulae for the eigenvalues that specify the decay rates of the perturbations. In this paper, we extend the analysis in axisymmetric geometry to the case where the disturbance depends on both the radial and azimuthal coordinates. Using a combination of analytical, asymptotic and numerical techniques, we show that all the disturbance eigenfunctions decay in time. We find that rates of decay are functions of the Froude number and azimuthal dependence. Perturbations of axisymmetric shape are shown to decay less rapidly than asymmetric perturbations in general. An asymptotic formula derived for the eigenvalues of the problem reveals that asymptotic rates of decay approach a constant value which depends on the Froude number only. The asymptotic rates of decay of perturbations are given by $t^{-\sigma}$, where $0<\sigma<\frac{1}{4}$, as the Froude number decreases from $\sqrt{2}$ to 0 . We demonstrate that the asymptotic formula has excellent convergence to numerically calculated values and, in the absence of azimuthal dependence, it simplifies to an expression that improves on the asymptotic formula 
presented by GR. We show how the eigenfunctions of the disturbance are related to the group of transformations under the action of which the problem is invariant. In twodimensional (planar) geometry, GR have found through numerical and asymptotic techniques that all the eigenfunctions of the symmetric perturbations decay like $t^{-1 / 2}$. We present an analytical proof of this result.

We present the mathematical formulation and self-similar solution of the problem in axisymmetric geometry in $\S 2$. A linear stability analysis is carried out in $\S 3$ to determine whether small perturbations involving both radial and azimuthal dependences, imposed on the self-similar solution, grow or decay in time. The asymptotic formula for the eigenvalues is also derived in $\$ 3.2$. In $\S 3.3$, we demonstrate the connection between the disturbance eigenfunctions and the symmetry transformations of the problem. The main findings of the paper are summarized in $\S 4$. The proof that all two-dimensional eigenfunctions decay like $t^{-1 / 2}$ is presented in the Appendix.

\section{Governing equations and self-similar solution}

We study the motion of a gravity current generated by the finite release from a localized source of a homogeneous fluid of density $\rho+\Delta \rho$ within a deep and quiescent ambient of constant density $\rho$. We adopt cylindrical polar coordinates $r, \theta, z$, and denote the vertically averaged velocity component in the radial direction by $u$. We assume that the horizontal lengthscale of the flow far exceeds the vertical lengthscale so that the pressure adopts a hydrostatic distribution. Furthermore, we neglect drag forces and assume that the azimuthal velocity component is identically zero. If the height of the current is denoted by $h$, the dimensionless shallow-water governing equations are given by

$$
\begin{aligned}
r \partial_{t} h+\partial_{r}(r u h) & =0, \\
\partial_{t} u+\partial_{r}\left(\frac{1}{2} u^{2}+h\right) & =0,
\end{aligned}
$$

representing conservation of mass and momentum, respectively, in the domain $0 \leqslant$ $r \leqslant r_{F}$, where $r_{F}(t)$ is the dimensionless position of the front. Here, lengths and times have been made dimensionless with respect to the lengthscale $h_{0}$ and the timescale $\left(h_{0} / g^{\prime}\right)^{1 / 2}$, respectively, where $h_{0}$ is the characteristic initial lengthscale of the current and $g^{\prime}=\Delta \rho g / \rho$ is the reduced gravity. We note that the same governing equations also apply to the flow of a gravity current in a sector of constant angle (Bonnecaze et al. 1995). The boundary conditions are given by

$$
\begin{aligned}
\int_{0}^{r_{F}} r h(r, t) \mathrm{d} r & =V, \\
u(0, t) & =0, \\
\dot{r}_{F}^{2} & =F r^{2} h\left(r_{F}, t\right), \\
\dot{r}_{F} & =u\left(r_{F}, t\right),
\end{aligned}
$$

where $2 \pi V$ is the dimensionless volume of the current, $\dot{r}=\mathrm{d} r / \mathrm{d} t$, and $F r$ denotes the Froude number. These equations represent the integral expression for global conservation of volume (2.3), symmetry condition at the origin (2.4), the dynamic condition at the front (2.5), and the kinematic condition at the front (2.6).

In (2.5), the Froude number provides a connection between the height and velocity at the front of the flow and is required to supplement the shallow-water model because, at the front, the motion may no longer be governed by hydrostatic balance. Previous theoretical and experimental analyses have determined the Froude number 
as a function of the height of the current relative to the depth of the flow (see, for example, Benjamin 1968; Huppert \& Simpson 1980; Shin et al. 2004). In this study, we consider a regime in which the current is moving through a much deeper ambient and the Froude number adopts a constant value; Benjamin (1968) determined that $F r=\sqrt{2}$ from theoretical considerations, while Huppert \& Simpson (1980) find experimentally that $F r=1.2$. Here we solely assume that $F r$ is a constant, but at this stage do not need to prescribe a particular value to develop the ensuing stability analysis.

The equations (2.1) and (2.2) prompt the scalings $u \sim r / t$ and $h \sim(r / t)^{2}$, respectively. These, when combined with the scaling $r^{2} h \sim V$ from (2.3), give the scale of the radius of the current $r \sim\left(V t^{2}\right)^{1 / 4}$. Motivated by these scales, we look for a self-similar solution of the form

$$
\begin{aligned}
& u(r, t)=\frac{1}{2} k t^{-1 / 2} U_{0}(\xi), \\
& h(r, t)=\frac{1}{4} k^{2} t^{-1} H_{0}(\xi),
\end{aligned}
$$

where the similarity variable $\xi=r /\left[b\left(V t^{2}\right)^{1 / 4}\right]$ and the constants $k$ and $b=k V^{-1 / 4}$ are to be determined such that $\xi=1$ at the front. Substituting into the system (2.1)-(2.6), and solving, yields (Fannelop \& Waldman 1972; Hoult 1972)

$$
\begin{aligned}
& U_{0}(\xi)=\xi, \\
& H_{0}(\xi)=\frac{1}{2}\left(\xi^{2}+\ell\right),
\end{aligned}
$$

where $\ell=2 / F r^{2}-1$ and the constant $b=\left[32 F r^{2} /\left(4-F r^{2}\right)\right]^{1 / 4}$. The position of the front is given by $r_{F}=k t^{1 / 2}$. This similarity solution is valid for Froude numbers in the interval $0<F r \leqslant \sqrt{2}$. As the Froude number increases (with the volume of the current kept fixed), the length of the current increases while its height at the origin decreases. When $F r=\sqrt{2}$, we have $b=2^{5 / 4}, \ell=0, H_{0}(\xi)=\frac{1}{2} \xi^{2}$, and the height of the current vanishes at the origin.

When $F r>\sqrt{2}$, the parameter $\ell=\left(2 / F r^{2}-1\right)<0$ and the self-similar height profile is given by

$$
H_{0}(\xi)= \begin{cases}0, & 0<\xi<\xi_{c}, \\ \frac{1}{2}\left(\xi^{2}+\ell\right), & \xi_{c}<\xi<1,\end{cases}
$$

where $\xi_{c}=\left(1-2 / F r^{2}\right)^{1 / 2}>0$. In this case, no fluid occupies the region $0<\xi<\xi_{c}$ around the origin, and the constant that determines the position of the front of the current is given by $b=2^{3 / 4} \mathrm{Fr}$.

We remark that the analysis carried out in the remainder of this paper focuses on the similarity solution (2.7)-(2.10) valid for $0<F r \leqslant \sqrt{2}$. It has been shown by GR that a small radially symmetric disturbance imposed on this solution decays in time. The purpose of the present study is to analyse the case where the imposed disturbance depends on both the radial and azimuthal coordinates.

\section{3. $\theta$-dependent perturbation}

We study the evolution of the self-similar solution (2.7)-(2.10) subsequent to the introduction of a small disturbance that depends on both the radial and the azimuthal coordinates. The (dimensionless) governing equations, which must also account for azimuthal variations now, are given by

$$
\begin{aligned}
r \partial_{t} h+\partial_{r}(r u h)+\partial_{\theta}(v h) & =0, \\
r \partial_{t} u+r \partial_{r}\left(\frac{1}{2} u^{2}+h\right)+v \partial_{\theta} u-v^{2} & =0, \\
r \partial_{t} v+r u \partial_{r} v+u v+v \partial_{\theta} v+\partial_{\theta} h & =0,
\end{aligned}
$$


where $v$ represents the depth-averaged velocity component in the azimuthal direction. These equations are expressions of conservation of mass (3.11), radial component of conservation of momentum (3.12), and azimuthal component of conservation of momentum (3.13). They are valid in the domain $0 \leqslant r \leqslant r_{F}$, where $r_{F}(\theta, t)$ is the dimensionless position of the front, and are subject to the following boundary conditions.

The expression for global conservation of volume now becomes

$$
\int_{0}^{2 \pi} \int_{0}^{r_{F}} r h(r, \theta, t) \mathrm{d} r \mathrm{~d} \theta=2 \pi V .
$$

At the origin, the symmetry condition no longer holds, but we must have finite flux and height.

We define the position of the front as $\mathscr{F}(r, \theta, t)=r-r_{F}(\theta, t)=0$. Thus $\nabla \mathscr{F}=$ $\left(r_{F} \hat{\boldsymbol{r}}-r_{F}^{\prime} \hat{\boldsymbol{\theta}}\right) / r_{F}$ and the normal velocity of the front is given by

$$
-\frac{\partial_{t} \mathscr{F}}{|\nabla \mathscr{F}|}=-\frac{\dot{r}_{F} r_{F}}{\sqrt{r_{F}^{2}+{r_{F}^{\prime}}^{2}}},
$$

where $r_{F}^{\prime}=\partial_{\theta} r_{F}, \dot{r}_{F}=\partial_{t} r_{F}$, and $\hat{\boldsymbol{r}}$ and $\hat{\boldsymbol{\theta}}$ are unit vectors in the radial and azimuthal directions, respectively. The normal velocity of the fluid at the front is

$$
\boldsymbol{u} \cdot \hat{\boldsymbol{n}}=\boldsymbol{u} \cdot \frac{\nabla \mathscr{F}}{|\nabla \mathscr{F}|}=\frac{u r_{F}-v r_{F}^{\prime}}{\sqrt{r_{F}^{2}+r_{F}^{\prime 2}}},
$$

where $\boldsymbol{u}=u \hat{\boldsymbol{r}}+v \hat{\boldsymbol{\theta}}$. Since the flux across the front is zero, the curve $\mathscr{F}=0$ must always be composed of the same fluid particles, and hence (3.15) is equal to (3.16). This yields the kinematic condition

$$
r_{F} \dot{r}_{F}+r_{F}^{\prime} v=r_{F} u, \quad \text { at } r=r_{F} .
$$

The Froude number condition in this case becomes $\boldsymbol{u} \cdot \hat{\boldsymbol{n}}=\operatorname{Fr}\left\{h\left(r_{F}, \theta, t\right)\right\}^{1 / 2}$, which gives

$$
r_{F} u-r_{F}^{\prime} v=F r\left(r_{F}^{2}+r_{F}^{\prime 2}\right)^{1 / 2} h^{1 / 2}, \quad \text { at } r=r_{F} .
$$

\subsection{Linear stability analysis}

To analyse the evolution of the perturbation, we introduce expansions about the self-similar solution as follows:

$$
\begin{aligned}
u(r, \theta, t) & =\frac{1}{2} k t^{-1 / 2}\left\{U_{0}(\xi)+\delta U_{1}(\xi, \theta, t)+\cdots\right\}, \\
v(r, \theta, t) & =\frac{1}{2} k t^{-1 / 2}\left\{\delta V_{1}(\xi, \theta, t)+\cdots\right\}, \\
h(r, \theta, t) & =\frac{1}{4} k^{2} t^{-1}\left\{H_{0}(\xi)+\delta H_{1}(\xi, \theta, t)+\cdots\right\}, \\
r_{F}(\theta, t) & =k t^{1 / 2}\left\{1+\delta \xi_{F 1}(\theta, t)+\cdots\right\},
\end{aligned}
$$

where $\delta \ll 1$ is the amplitude of the perturbations. Substituting these expansions into the governing equations (3.11)-(3.18) recovers the self-similar solution at $O(1)$. At $O(\delta)$, the equations governing the evolution of the perturbation quantities are found to be

$$
\begin{aligned}
2 \xi t \partial_{t} H_{1}+\partial_{\xi}\left(\xi H_{0} U_{1}\right)+H_{0} \partial_{\theta} V_{1}=0 \\
2 t \partial_{t} U_{1}+\partial_{\xi} H_{1}=0 \\
2 \xi t \partial_{t} V_{1}+\partial_{\theta} H_{1}=0
\end{aligned}
$$


The boundary conditions at the front of the current become

$$
\begin{aligned}
& U_{1}(1, \theta, t)=2 t \dot{\xi}_{F 1}, \\
& H_{1}(1, \theta, t)=2(\ell+1) t \dot{\xi}_{F 1}+\ell \xi_{F 1} .
\end{aligned}
$$

At the origin, $H_{1}, U_{1}$, and $V_{1}$ must remain finite.

The equations (3.19)-(3.21) can be combined into the following second-order partial differential equation for $H_{1}$ :

$$
4 \xi^{2} t \partial_{t}\left(t \partial_{t} H_{1}\right)=\xi \partial_{\xi}\left(\xi H_{0} \partial_{\xi} H_{1}\right)+H_{0} \partial_{\theta} \partial_{\theta} H_{1},
$$

subject to the boundary condition (3.23) at the front and that $H_{1}$ must remain finite at the origin. A third boundary condition for $H_{1}$ is obtained by combining (3.20) and (3.22), and it is given by

$$
\partial_{\xi} H_{1}(1, \theta, t)=-4\left(t \dot{\xi}_{F 1}+t^{2} \ddot{\xi}_{F 1}\right) .
$$

By the method of separation of variables we then look for a solution of the form

$$
\begin{aligned}
H_{1}(\xi, \theta, t) & =\sum_{m=0}^{\infty} \sum_{n=0}^{\infty} \alpha_{m n} t^{\lambda_{m n}} \mathrm{e}^{\mathrm{i} m \theta} \Phi_{m n}(\xi), \\
\xi_{F 1}(\theta, t) & =\sum_{m=0}^{\infty} \sum_{n=0}^{\infty} \gamma_{m n} t^{\lambda_{m n}} \mathrm{e}^{\mathrm{i} m \theta},
\end{aligned}
$$

where $\alpha_{m n}$ and $\gamma_{m n}$ are complex constants, and $\lambda_{m n}=\Lambda_{m n}+\mathrm{i} \Omega_{m n}$ (with $\Lambda_{m n}, \Omega_{m n} \in \mathbb{R}$ ) are the eigenvalues of the system. Proving linear stability of the self-similar solution (2.7)-(2.10) therefore involves showing that none of the $\Lambda_{m n}$ are positive. Substituting into the system (3.22)-(3.25) indicates that the functions $\Phi_{m n}$ satisfy the following ordinary differential equation:

$$
\xi^{2}\left(\xi^{2}+\ell\right) \Phi_{m n}^{\prime \prime}+\xi\left(3 \xi^{2}+\ell\right) \Phi_{m n}^{\prime}-\left\{\left(8 \lambda^{2}+m^{2}\right) \xi^{2}+m^{2} \ell\right\} \Phi_{m n}=0,
$$

subject to the boundary conditions

$$
\begin{aligned}
& \Phi_{m n}(1)=\gamma_{m n}\left[2(\ell+1) \lambda_{m n}+\ell\right] / \alpha_{m n}, \\
& \Phi_{m n}^{\prime}(1)=-4 \gamma_{m n} \lambda_{m n}^{2} / \alpha_{m n}, \\
& \Phi_{m n}(0) \text { is non-singular. }
\end{aligned}
$$

Making the substitutions $\xi^{2}=-\ell \eta$ and $\Phi_{m n}=\eta^{m / 2} G_{m n}(\eta)$, successively, transforms equation (3.26) into

$$
\eta(1-\eta) G_{m n}^{\prime \prime}+\{1+m-(2+m) \eta\} G_{m n}^{\prime}+\left(2 \lambda_{m n}^{2}-\frac{1}{2} m\right) G_{m n}=0,
$$

which is the hypergeometric differential equation (Abramowitz \& Stegun 1965). A solution which satisfies (3.27) and (3.29) is found to be

$$
\Phi_{m n}(\xi)=\gamma_{m n}\left[2(\ell+1) \lambda_{m n}+\ell\right] \xi^{m} \frac{{ }_{2} F_{1}\left(a_{m n}, b_{m n} ; m+1 ;-\xi^{2} / \ell\right)}{\alpha_{m n}{ }_{2} F_{1}\left(a_{m n}, b_{m n} ; m+1 ;-1 / \ell\right)},
$$

where

$$
\begin{aligned}
& a_{m n}=\frac{1}{2}(m+1)+\frac{1}{2} \sqrt{1+m^{2}+8 \lambda_{m n}^{2}}, \\
& b_{m n}=\frac{1}{2}(m+1)-\frac{1}{2} \sqrt{1+m^{2}+8 \lambda_{m n}^{2}},
\end{aligned}
$$

and the symbol ${ }_{2} F_{1}$ denotes the hypergeometric function. 
At this stage, we can show that all $\Lambda_{m n}<0$. Using an approach similar to that employed by GR, we take the complex conjugate of (3.26), multiply by $\lambda_{m n}^{2} \Phi_{m n}$, and integrate over $[0,1]$ to get

$$
\begin{array}{r}
\lambda_{m n}^{2} \int_{0}^{1} \Phi_{m n}\left[\xi\left(\xi^{2}+\ell\right) \bar{\Phi}_{m n}^{\prime}\right]^{\prime} \mathrm{d} \xi-8\left|\lambda_{m n}^{2}\right|^{2} \int_{0}^{1} \xi\left|\Phi_{m n}\right|^{2} \mathrm{~d} \xi \\
-m^{2} \lambda_{m n}^{2} \int_{0}^{1}\left(\xi+\frac{\ell}{\xi}\right)\left|\Phi_{m n}\right|^{2} \mathrm{~d} \xi=0
\end{array}
$$

where $\bar{z}$ denotes the complex conjugate of $z$. Evaluating the first integral by parts and using the boundary conditions (3.27)-(3.29) yields a quadratic equation for $\lambda_{m n}$,

$$
\mathscr{A} \lambda_{m n}^{2}+\mathscr{B} \lambda_{m n}+\mathscr{C}=0
$$

where

$$
\begin{aligned}
& \mathscr{A}=\int_{0}^{1}\left\{m^{2}\left|\Phi_{m n}\right|^{2}+\xi^{2}\left|\Phi_{m n}^{\prime}\right|^{2}\right\}\left(\frac{\xi^{2}+\ell}{\xi}\right) \mathrm{d} \xi \\
& \mathscr{B}=8(1+\ell)^{2}\left|\frac{\lambda_{m n}^{2} \gamma_{m n}}{\alpha_{m n}}\right|^{2}, \\
& \mathscr{C}=4 \ell(1+\ell)\left|\frac{\lambda_{m n}^{2} \gamma_{m n}}{\alpha_{m n}}\right|^{2}+8\left|\lambda_{m n}^{2}\right|^{2} \int_{0}^{1} \xi\left|\Phi_{m n}\right|^{2} \mathrm{~d} \xi .
\end{aligned}
$$

The term $\mathscr{A}$ is regular at $\xi=0$ only if $\left|\Phi_{m n}\right|^{2}=O\left(\xi^{\varphi}\right)$, where $\varphi>0$. From (3.31) we have $\left|\Phi_{m n}\right|^{2}=O\left(\xi^{2 m}\right)$ as $\xi \rightarrow 0$, which implies that $\mathscr{A}$ will be non-singular when $m>0$. However, $\mathscr{A}$ is also non-singular when $m=0$ because the term involving $1 / \xi$ in the integrand vanishes. Thus, $\mathscr{A}$ is positive and real for all $\lambda_{m n}$. Both $\mathscr{B}$ and $\mathscr{C}$ are also real and positive for all $\lambda_{m n}$ and hence equation (3.33) satisfied by the eigenvalues $\lambda_{m n}$ has real positive coefficients. This has two implications: first, all eigenvalues $\lambda_{m n}$ must occur in complex conjugate pairs and, second, all eigenvalues $\lambda_{m n}$ must have negative real parts. The latter establishes that all perturbation eigenfunctions decay in time and hence the self-similar solution (2.7)-(2.10) is linearly stable to perturbations which depend on both $r$ and $\theta$.

\subsection{Asymptotic formula for eigenvalues}

The eigenvalues are found by requiring that the solution (3.31) should also satisfy the boundary condition (3.28). Substituting (3.31) into (3.28) gives the following equation for the eigenvalues $\lambda_{m n}$

$$
\frac{{ }_{2} F_{1}\left(a_{m n}+1, b_{m n}+1 ; m+2 ;-1 / \ell\right)}{{ }_{2} F_{1}\left(a_{m n}, b_{m n} ; m+1 ;-1 / \ell\right)}=\frac{-\ell(m+1)}{4 \lambda_{m n}^{2}-m}\left\{m+\frac{4 \lambda_{m n}^{2}}{2(\ell+1) \lambda_{m n}+\ell}\right\} .
$$

We remark that there exist simple eigensolutions of (3.26) for which (3.34) is trivially satisfied and they correspond to the eigenvalues $\lambda_{0 V}=0, \lambda_{0 T}=-1$ for $m=0$, and $\lambda_{1 S}=-\frac{1}{2}$ for $m=1$. We demonstrate below that these eigensolutions are associated with volume-changing, time-shifting and space-translating transformations, respectively. Thus the eigenvalue $\lambda_{O V}=0$ does not negate the stability. 


\begin{tabular}{|c|c|c|c|c|c|c|}
\hline & \multicolumn{6}{|c|}{$m=0$} \\
\hline & Numerical $\lambda_{0 n}$ & \multicolumn{2}{|c|}{ Asymptotic $\lambda_{0 n}$} & Absolut & error & $\%$ Error \\
\hline & $-0.1966+1.4717 \mathrm{i}$ & \multicolumn{2}{|c|}{$-0.2203+\left\{\begin{array}{l}1.5753 \mathrm{i} \\
1.2602 \mathrm{i}(\mathrm{GR})\end{array}\right.$} & & & $\begin{array}{r}7.0 \\
14.4\end{array}$ \\
\hline & $-0.2134+2.7768 \mathrm{i}$ & \multicolumn{2}{|c|}{$-0.2203+\left\{\begin{array}{l}2.8355 \mathrm{i} \\
2.5204 \mathrm{i}(\mathrm{GR})\end{array}\right.$} & & & $\begin{array}{l}2.1 \\
9.2\end{array}$ \\
\hline & $-0.2171+4.0550 \mathrm{i}$ & \multicolumn{2}{|c|}{$-0.2203+\left\{\begin{array}{l}4.0957 \mathrm{i} \\
3.7806 \mathrm{i}(\mathrm{GR})\end{array}\right.$} & & & $\begin{array}{l}1.0 \\
6.8\end{array}$ \\
\hline & $-0.2184+5.3248 \mathrm{i}$ & \multicolumn{2}{|c|}{$-0.2203+\left\{\begin{array}{l}5.3559 \mathrm{i} \\
5.0409 \mathrm{i}(\mathrm{GR})\end{array}\right.$} & & & $\begin{array}{l}0.6 \\
5.3\end{array}$ \\
\hline & $-0.2191+6.5909 i$ & \multicolumn{2}{|c|}{$-0.2203+\left\{\begin{array}{l}6.6161 \mathrm{i} \\
6.3011 \mathrm{i}(\mathrm{GR})\end{array}\right.$} & & & $\begin{array}{l}0.4 \\
4.4\end{array}$ \\
\hline & $-0.2195+7.8551 \mathrm{i}$ & \multicolumn{2}{|c|}{$-0.2203+\left\{\begin{array}{l}7.8763 \mathrm{i}^{7.5613 \mathrm{i}(\mathrm{GR})} \\
7.0\end{array}\right.$} & & & $\begin{array}{l}0.3 \\
3.7\end{array}$ \\
\hline & $-0.2197+9.1183 i$ & \multicolumn{2}{|c|}{$-0.2203+\left\{\begin{array}{l}9.1366 \mathrm{i} \\
8.8215 \mathrm{i}(\mathrm{GR})\end{array}\right.$} & & & $\begin{array}{l}0.2 \\
3.3\end{array}$ \\
\hline & $-0.2198+10.3807 i$ & \multicolumn{2}{|c|}{$-0.2203+\left\{\begin{array}{l}10.3968 \mathrm{i} \\
10.0817 \mathrm{i}(\mathrm{GR})\end{array}\right.$} & & & $\begin{array}{l}0.2 \\
2.9\end{array}$ \\
\hline & $-0.2199+11.6426 \mathrm{i}$ & \multicolumn{2}{|c|}{$-0.2203+\left\{\begin{array}{l}11.6570 \mathrm{i} \\
11.3419 \mathrm{i}(\mathrm{GR})\end{array}\right.$} & & & $\begin{array}{l}0.1 \\
2.6\end{array}$ \\
\hline \multirow[t]{2}{*}{10} & $-0.2200+12.9043 \mathrm{i}$ & \multicolumn{2}{|c|}{$-0.2203+\left\{\begin{array}{l}12.9172 \mathrm{i} \\
12.6022 \mathrm{i}(\mathrm{GR})\end{array}\right.$} & & & $\begin{array}{l}0.0 \\
2.3\end{array}$ \\
\hline & \multicolumn{2}{|c|}{$m=1$} & \multicolumn{4}{|c|}{$m=2$} \\
\hline & Numerical $\lambda_{m n}$ & Asymptotic $\lambda_{m n}$ & & cal $\lambda_{m n}$ & $\mathrm{~A}$ & tic $\lambda_{m n}$ \\
\hline & $\begin{array}{l}-0.2176+2.1059 \mathrm{i} \\
-0.2193+3.4040 \mathrm{i} \\
-0.2198+4.6818 \mathrm{i} \\
-0.2200+5.9515 \mathrm{i} \\
-0.2201+7.2178 \mathrm{i} \\
-0.2202+8.4822 \mathrm{i} \\
-0.2202+9.7456 \mathrm{i} \\
-0.2202+11.0082 \mathrm{i} \\
-0.2203+12.2704 \mathrm{i} \\
-0.2203+13.5322 \mathrm{i}\end{array}$ & $\begin{array}{l}-\Lambda_{\infty}+2.2054 \mathrm{i} \\
-\Lambda_{\infty}+3.4656 \mathrm{i} \\
-\Lambda_{\infty}+4.7258 \mathrm{i} \\
-\Lambda_{\infty}+5.9860 \mathrm{i} \\
-\Lambda_{\infty}+7.2462 \mathrm{i} \\
-\Lambda_{\infty}+8.5065 \mathrm{i} \\
-\Lambda_{\infty}+9.7667 \mathrm{i} \\
-\Lambda_{\infty}+11.0269 \mathrm{i} \\
-\Lambda_{\infty}+12.2871 \mathrm{i} \\
-\Lambda_{\infty}+13.5473 \mathrm{i}\end{array}$ & $\begin{array}{l}-0.2 \\
-0.2 \\
-0.2 \\
-0.2 \\
-0.2 \\
-0.2 \\
-0.2 \\
-0.2 \\
-0.2 \\
-0.2\end{array}$ & $\begin{array}{l}5+2.7056 \mathrm{i} \\
9+4.0127 \mathrm{i} \\
5+5.2942 \mathrm{i} \\
0+6.5668 \mathrm{i} \\
2+7.8352 \mathrm{i} \\
7+9.1013 \mathrm{i} \\
+10.3658 \mathrm{i} \\
2+11.6295 \\
0+12.8924 \\
9+14.1549\end{array}$ & $\begin{array}{l}-\Lambda_{\infty} \\
-\Lambda_{\infty} \\
-\Lambda_{\infty} \\
-\Lambda_{\infty} \\
-\Lambda_{\infty} \\
-\Lambda_{\infty} \\
-\Lambda_{\infty} \\
-\Lambda_{\infty} \\
-\Lambda_{\infty} \\
-\Lambda_{\infty}\end{array}$ & $\begin{array}{l}-2.8355 \mathrm{i} \\
-4.0957 \mathrm{i} \\
-5.3559 \mathrm{i} \\
-6.6161 \mathrm{i} \\
-7.8763 \mathrm{i} \\
-9.1366 \mathrm{i} \\
-10.3968 \mathrm{i} \\
-11.6570 \mathrm{i} \\
-12.9172 \mathrm{i} \\
-14.1774 \mathrm{i}\end{array}$ \\
\hline
\end{tabular}

TABLE 1. The first ten eigenvalues for $m=0,1$, and $m=2$, respectively. For the case $m=0$, the asymptotic results of GR are also shown. In all cases, eigenvalues have been computed correct to four decimal places, $F r=1$, and $\Lambda_{\infty} \simeq 0.2203$. form

Using the formula (A 18) from the Appendix, we express equation (3.34) in the

$$
\begin{aligned}
& \left.\frac{{ }_{2} F_{1}\left(m-a_{m n}+1, m-b_{m n}+1 ; m+2 ;-1 / \ell\right)}{{ }_{2} F_{1}\left(m-a_{m n}+1, m-b_{m n}\right.}+1 ; m+1 ;-1 / \ell\right) \\
& =\frac{-(\ell+1)(m+1)}{4 \lambda_{m n}^{2}-m}\left\{m+\frac{4 \lambda_{m n}^{2}}{2(\ell+1) \lambda_{m n}+\ell}\right\} .
\end{aligned}
$$

We have solved this equation numerically using MAPLE and values of $\lambda_{m n}$ obtained are shown in table 1 . It is useful to also derive an asymptotic formula for the eigenvalues 
by taking the limit $\left|\Omega_{m n}\right| \rightarrow \infty$. We note that $\sqrt{1+m^{2}+8 \lambda_{m n}^{2}} \sim 2 \sqrt{2} \lambda_{m n}$ for $\Omega_{m n} \gg m$ and hence the leading-order terms in equation (3.35) may be written as

$$
\begin{aligned}
& \frac{{ }_{2} F_{1}\left(\frac{1}{2}(m+1)+\sqrt{2} \lambda_{m n}, \frac{1}{2}(m+1)-\sqrt{2} \lambda_{m n} ; m+2 ;-1 / \ell\right)}{{ }_{2} F_{1}\left(\frac{1}{2}(m+1)+\sqrt{2} \lambda_{m n}, \frac{1}{2}(m+1)-\sqrt{2} \lambda_{m n} ; m+1 ;-1 / \ell\right)} \\
& =\frac{-(\ell+1)(m+1)}{4 \lambda_{m n}^{2}-m}\left\{m+\frac{4 \lambda_{m n}^{2}}{2(\ell+1) \lambda_{m n}+\ell}\right\} .
\end{aligned}
$$

We express the hypergeometric functions in integral form and the left-hand side of (3.36) becomes

$$
\frac{2(m+1)}{m+1-2 \sqrt{2} \lambda_{m n}} \times \frac{\int_{0}^{1} \mathscr{T}^{\sqrt{2} \lambda_{m n}+\frac{m-1}{2}}(1-\mathscr{T})^{\frac{m+1}{2}-\sqrt{2} \lambda_{m n}}(\ell+\mathscr{T})^{\sqrt{2} \lambda_{m n}-\frac{m+1}{2}} \mathrm{~d} \mathscr{T}}{\int_{0}^{1} \mathscr{T}^{\sqrt{2} \lambda_{m n}+\frac{m-1}{2}}(1-\mathscr{T})^{\frac{m-1}{2}-\sqrt{2} \lambda_{m n}}(\ell+\mathscr{T})^{\sqrt{2} \lambda_{m n}-\frac{m+1}{2}} \mathrm{~d} \mathscr{T}} .
$$

It is convenient to make the substitution $\zeta=\mathscr{T} /(1-\mathscr{T})$ which transforms the integrals to

$$
\Xi=\frac{\int_{0}^{\infty} \zeta^{\sqrt{2} \lambda_{m n}+\frac{m-1}{2}}(1+\zeta)^{-\frac{m+3}{2}-\sqrt{2} \lambda_{m n}}[\ell+(1+\ell) \zeta]^{\sqrt{2} \lambda_{m n}-\frac{m+1}{2}} \mathrm{~d} \zeta}{\int_{0}^{\infty} \zeta^{\sqrt{2} \lambda_{m n}+\frac{m-1}{2}}(1+\zeta)^{-\frac{m+1}{2}-\sqrt{2} \lambda_{m n}}[\ell+(1+\ell) \zeta]^{\sqrt{2} \lambda_{m n}-\frac{m+1}{2}} \mathrm{~d} \zeta}
$$

The leading-order estimate of these integrals in the limit $\Omega_{m n} \rightarrow \infty$ can now be calculated using the method of steepest descents. We find

$$
\sqrt{\ell+1}\left\{\frac{\mathrm{i} p_{1} \mathrm{e}^{\mathrm{i} \vartheta_{1}}+p_{2} \mathrm{e}^{\mathrm{i} \vartheta_{2}}}{-\mathrm{i} p_{1} \mathrm{e}^{\mathrm{i} \vartheta_{1}}+p_{2} \mathrm{e}^{\mathrm{i} \vartheta_{2}}}\right\}
$$

where

$$
\begin{array}{ll}
p_{1}=(\sqrt{\ell+1}+1)^{2 \sqrt{2} \Lambda_{m n}}, & \vartheta_{1}=2 \sqrt{2} \Omega_{m n} \ln (\sqrt{\ell+1}+1)-\frac{1}{2} m \pi, \\
p_{2}=(\sqrt{\ell+1}-1)^{2 \sqrt{2} \Lambda_{m n},}, & \vartheta_{2}=2 \sqrt{2} \Omega_{m n} \ln (\sqrt{\ell+1}-1)+\frac{1}{2} m \pi .
\end{array}
$$

Equation (3.36) now becomes

$$
\Xi=\frac{(\ell+1)\left(2 \sqrt{2} \lambda_{m n}-m-1\right)}{2\left(4 \lambda_{m n}^{2}-m\right)}\left\{m+\frac{4 \lambda_{m n}^{2}}{2(\ell+1) \lambda_{m n}+\ell}\right\},
$$

and the expression on the right-hand side approaches $1 / \sqrt{2}$ as $\Omega_{m n} \rightarrow \infty$. Thus, in the limit $\Omega_{m n} \rightarrow \infty$, equation (3.36) becomes

$$
\sqrt{\ell+1}\left\{\frac{\mathrm{i} p_{1} \mathrm{e}^{\mathrm{i} \vartheta_{1}}+p_{2} \mathrm{e}^{\mathrm{i} \vartheta_{2}}}{-\mathrm{i} p_{1} \mathrm{e}^{\mathrm{i} \vartheta_{1}}+p_{2} \mathrm{e}^{\mathrm{i} \vartheta_{2}}}\right\}=\frac{1}{\sqrt{2}} .
$$

The imaginary components of (3.38) yield

$$
(\sqrt{\ell+1}+1)^{2 \sqrt{2} \Lambda_{m n}}(\sqrt{2(\ell+1)}+1) \cos \left(\vartheta_{1}-\vartheta_{2}\right)=0 .
$$

Since $\ell \geqslant 0$, because $\ell+1=2 / F r^{2}$ and $0<F r \leqslant \sqrt{2}$ for the self-similar solutions under consideration, the solution of this equation is $\cos \left(\vartheta_{1}-\vartheta_{2}\right)=0$, which gives

$$
\vartheta_{1}-\vartheta_{2}=\left(n+\frac{1}{2}\right) \pi, \quad n=0,1,2,3, \ldots
$$


Balancing the real parts of equation (3.38) then gives

$$
\left\{\frac{\sqrt{\ell+1}+1}{\sqrt{\ell+1}-1}\right\}^{2 \sqrt{2} \Lambda_{m n}}=(-1)^{n} \frac{\sqrt{2(\ell+1)}-1}{\sqrt{2(\ell+1)}+1},
$$

for which no solution exists when $n$ is odd. When $n$ is even the solution

$$
\Lambda_{m n}=\frac{-\{\ln (\sqrt{2(\ell+1)}+1)-\ln (\sqrt{2(\ell+1)}-1)\}}{2 \sqrt{2}\{\ln (\sqrt{\ell+1}+1)-\ln (\sqrt{\ell+1}-1)\}} .
$$

Equation (3.39) now gives

$$
\Omega_{m n}=\frac{\left(m+2 n+\frac{1}{2}\right) \pi}{2 \sqrt{2}\{\ln (\sqrt{\ell+1}+1)-\ln (\sqrt{\ell+1}-1)\}}, \quad m, n=0,1,2,3, \ldots
$$

where $n$ has been replaced by $2 n$ on the left-hand side because only even values are involved.

The values generated by expressions (3.40) and (3.41), derived by the foregoing asymptotic analysis, are expected to provide good estimates of $\lambda_{m n}$ in the regime $\Omega_{m n} \rightarrow \infty$. The asymptotic analysis however does not guarantee accuracy in the neighbourhood of $\Omega_{m n}=0$. A comparison of the eigenvalues generated by the asymptotic expressions with those calculated numerically is shown in table 1 and figure 1 . The rate of convergence between the asymptotic and numerical values is shown to be very high as $\Omega_{m n}$ increases. In the neighbourhood of $\Omega_{m n}=0$, our numerical calculations yielded no eigenvalues corresponding to the eigenvalues given by the asymptotic expressions when $n=0$. We conclude that these eigenvalues are spurious and exclude $n=0$.

Therefore, for large values of $\left|\Omega_{m n}\right|$, the eigenvalues are given by

$$
\lambda_{m n}=\frac{-\{\ln (2+F r)-\ln (2-F r)\} \pm \mathrm{i}\left(m+2 n+\frac{1}{2}\right) \pi}{2 \sqrt{2}\{\ln (\sqrt{2}+F r)-\ln (\sqrt{2}-F r)\}},
$$

where $m=0,1,2, \ldots$ and $n=1,2,3, \ldots$

Setting $m=0$ in (3.42) gives

$$
\lambda_{0 n}=\frac{-\{\ln (2+F r)-\ln (2-F r)\} \pm \mathrm{i}\left(2 n+\frac{1}{2}\right) \pi}{2 \sqrt{2}\{\ln (\sqrt{2}+F r)-\ln (\sqrt{2}-F r)\}}, \quad n=1,2,3, \ldots,
$$

which is an asymptotic expression for the eigenvalues of axisymmetric perturbations. This formula differs from the asymptotic result presented by GR (after we have corrected a typographical error of a factor of 2 in their formula) by an additive constant of $\frac{1}{2} \mathrm{i} \pi$ in the numerator. We demonstrate below that the estimates generated by (3.43) are superior to those generated by the formula of GR.

The perturbation height function is given by

$$
H_{1}=\sum_{m=0}^{\infty} \sum_{n=0}^{\infty} t^{\Lambda_{m n}} \Phi_{m n}(\xi)\left\{c_{m n} \cos \left(m \theta+\Omega_{m n} \ln t\right)+d_{m n} \sin \left(m \theta+\Omega_{m n} \ln t\right)\right\},
$$

where $c_{m n}$ and $d_{m n}$ are constants related to $\alpha_{m n}$ and $\gamma_{m n}$ and their values are determined from the initial distribution of the disturbance. The form of the eigenfunctions $\Phi_{m n}$ is shown in figure 2. It is shown that at the origin, in the absence of $\theta$-dependence, all the height perturbation eigenfunctions are non-zero but they have zero gradients. The presence of $\theta$-dependence means the symmetry at the origin is broken and then all the height perturbation eigenfunctions vanish at the origin. 


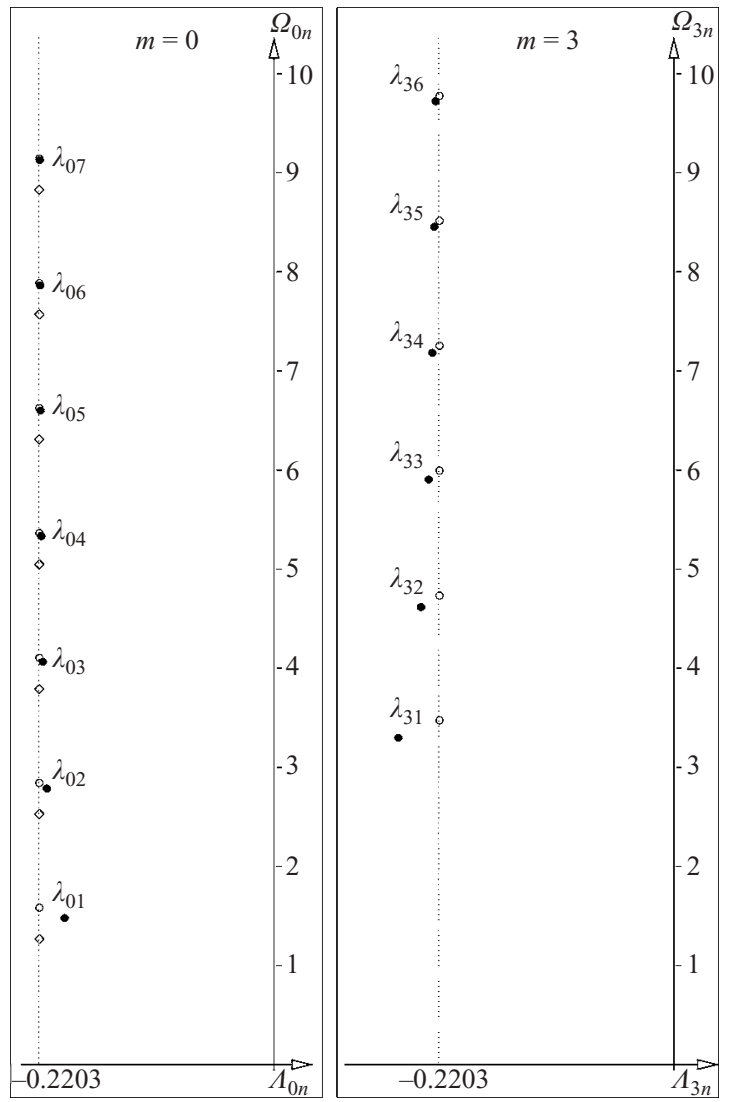

FiguRE 1. A comparison of the eigenvalues calculated numerically $(\bullet)$ and using the asymptotic formula (3.42) (O) for $m=0$ and $m=3$, respectively. In both cases $F r=1$. For the case $m=0$, the predictions of the asymptotic formula of GR $(\diamond)$ are also displayed for comparison.

For $m=0$, our numerically calculated eigenvalues are in agreement with those obtained by GR (for $\beta=1$ in their notation). A comparison of the estimates provided by the asymptotic formula (3.43) and the asymptotic formula of GR is shown in table 1 and figure 1 . While both formulas predict the same spacing between consecutive values of $\Omega_{0 n}$, our formula is shown to produce a better estimate even for the smallest $\Omega_{0 n}$, and also has a faster rate of convergence towards the exact eigenvalues as $\Omega_{0 n}$ increases. In fact, even though the relative errors for both formulas decrease as $\Omega_{0 n}$ increases, the absolute error for the formula of GR is shown to increase as $\Omega_{0 n}$ increases. This means that the distance between the estimates and the exact eigenvalues does not become smaller as $\Omega_{0 n}$ increases.

For general values of $m$, the asymptotic analysis indicates that as $\Omega_{m n} \rightarrow \infty$ keeping $F r$ fixed, the rates of decay, $\Lambda_{m n}$, of the eigenfunctions approach the same value (given by (3.40) and denoted by $\Lambda_{\infty}$ hereafter) irrespective of whether the disturbance depends on $\theta$ or not. A graphical illustration of how $\Lambda_{\infty}$ varies with $F r$ is shown in figure 3. It indicates that the asymptotic rates of decay of perturbations decrease from $t^{-1 / 4}$ towards $t^{0}$ as $F r$ increases from 0 to $\sqrt{2}$. For example, when $F r=1$, the asymptotic rate of decay is $t^{-0.22}$. The numerical calculations show that the direction from which the asymptotic rate of decay is approached is a function of $m$. For $m=0$ and $m=1$, 

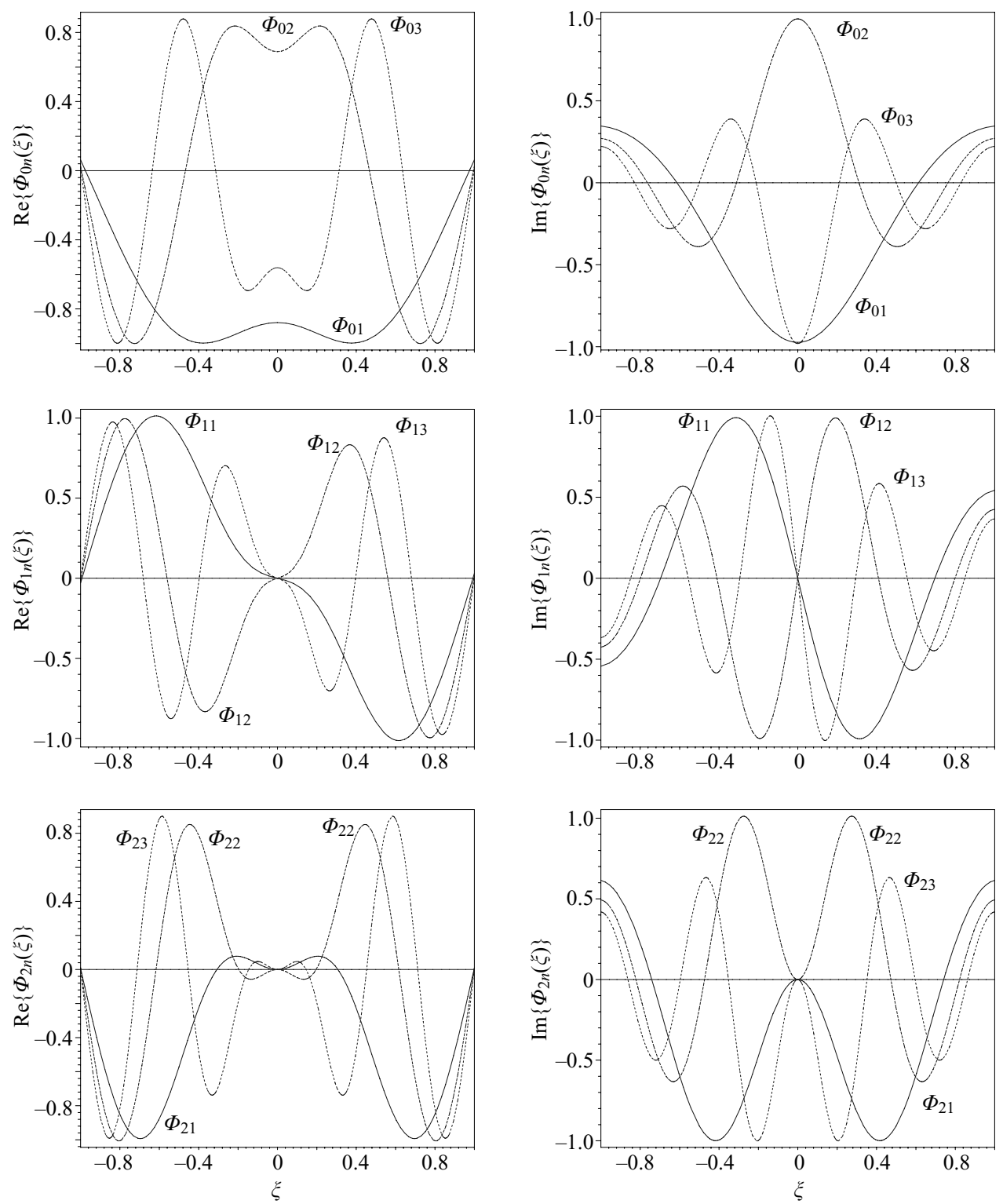

FIGURE 2. The first nine eigenfunctions of the perturbation height $H_{1}$ plotted as functions of the radial similarity variable $\xi$, calculated for $F r=1$. The positive and negative $\xi$-axes represent the $\theta=0$ and $\theta=\pi$ directions, respectively. All functions have been rescaled such that $\max \left\{\Phi_{m n}(\xi)\right\}=1$.

the direction of approach is from above, while for $m \geqslant 2$ the direction of approach is from below, as shown in figure 1. This represents a significant deviation from the usual situation whereby the eigenfunction corresponding to the smallest value of $n$ has the smallest rate of decay and hence gives the leading-order estimate of the disturbance. In this problem we can see that if the initial disturbance does not involve the $m=0$ and $m=1$ eigenfunctions of $\theta$, then the $\lambda_{m 1}$ eigenfunction will decay faster than all the subsequent eigenfunctions. For example, if the initial disturbance has the form $f_{0}(\xi) \cos 4 \theta$, then the first eigenfunction will decay approximately like $t^{-0.28}$ 


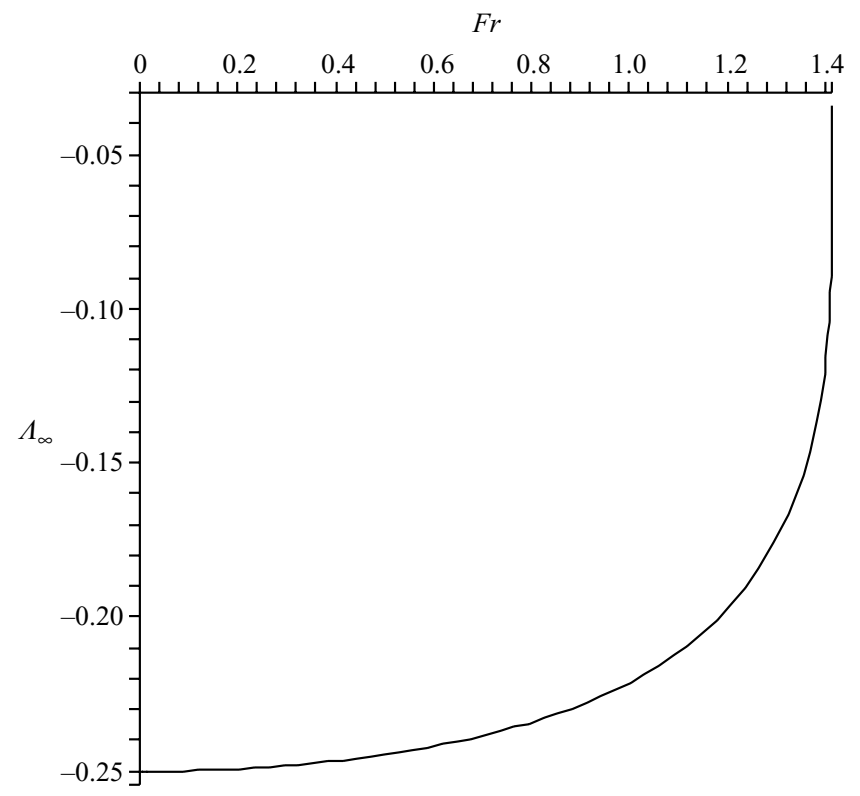

FIGURE 3 . The asymptotic rate of decay $\Lambda_{\infty}$ of perturbations plotted as a function of the Froude number $F r$.

while the second one decays like $t^{-0.25}$. The fact that $\Lambda_{\infty}$ is approached from below if $m \geqslant 2$ and from above otherwise also means that the smallest values of $\left|\Lambda_{m n}\right|$, and hence the lowest rates of decay, occur when $m=0$. Thus, axisymmetric perturbations will decay less rapidly than asymmetric ones in general. This is opposite to the result obtained for perturbations imposed on the axisymmetric Barenblatt-Pattle solution, where it has been shown (Mathunjwa \& Hogg 2006) that axisymmetric perturbations decay more rapidly than asymmetric ones.

\subsection{Connection with symmetry transformations}

We remark that the structure of the self-similar solution (2.7)-(2.10) is unchanged under the action of transformations that alter the volume of the current or shift the origin in time or space. We demonstrate that, for $m=0$, the simple eigenvalues $\lambda_{0 V}=0$ and $\lambda_{0 T}=-1$ are associated with volume-changing and time-shifting symmetry transformations, respectively. Substituting these eigenvalues into (3.31) and using (3.20) yields the eigenfunctions

$$
\begin{aligned}
& \Phi_{0 V}=\gamma_{0 V} \ell / \alpha_{0 V}, \\
& \Psi_{0 V}=0, \\
& \Phi_{0 T}=-\gamma_{0 T}\left(2 \xi^{2}+\ell\right) / \alpha_{0 T}, \\
& \Psi_{0 T}=-2 \gamma_{0 T} \xi / \alpha_{0 T} .
\end{aligned}
$$

To find the coefficients of infinitesimal transformations, we first express the similarity solution in terms of the primitive variables

$$
\begin{aligned}
& u(r, t)=\frac{1}{2} k t^{-1 / 2} U_{0}(\xi)=\frac{r}{2 t}, \\
& h(r, t)=\frac{1}{4} k^{2} t^{-1} H_{0}(\xi)=\frac{1}{8}\left\{\left(\frac{r}{t}\right)^{2}+\frac{k^{2} \ell}{t}\right\} .
\end{aligned}
$$


Transformations that alter the volume are represented by $V \longmapsto V+\delta$. Using the relation $k=b V^{1 / 4}$, the image of the similarity solution under the action of these transformations is given by

$$
\begin{aligned}
& u_{\delta}(r, t ; \delta)=u=\frac{1}{2} k t^{-1 / 2}\left\{U_{0}(\xi)+0\right\} \\
& h_{\delta}(r, t ; \delta)=h+\delta \frac{b^{2} \ell}{16 V^{1 / 2} t}=\frac{k^{2}}{4 t}\left\{H_{0}(\xi)+\delta \frac{\ell}{4 V}\right\} .
\end{aligned}
$$

This implies that the coefficients of the infinitesimal transformations $\left[\partial u_{\delta} / \partial \delta\right]_{\delta=0}=0$ and $\left[\partial h_{\delta} / \partial \delta\right]_{\delta=0}=$ constant, corresponding to the eigenfunctions found for $\lambda_{0 V}=0$.

Time-shift transformations are characterized by $t \longmapsto t+\delta$, and the image of the similarity solution is given by

$$
\begin{aligned}
& u_{\delta}=\frac{r}{2(t+\delta)}=\frac{k}{2 t^{1 / 2}}\left\{U_{0}(\xi)-\delta \frac{\xi}{t}\right\}, \\
& h_{\delta}=\frac{1}{8}\left\{\frac{r^{2}}{(t+\delta)^{2}}+\frac{k^{2} \ell}{(t+\delta)}\right\}=\frac{k^{2}}{4 t}\left\{H_{0}(\xi)-\frac{\delta}{2 t}\left(2 \xi^{2}+\ell\right)\right\} .
\end{aligned}
$$

Thus we have $\left[\partial u_{\delta} / \partial \delta\right]_{\delta=0}=-\xi / t$ and $\left[\partial h_{\delta} / \partial \delta\right]_{\delta=0}=-\frac{1}{2}\left(2 \xi^{2}+\ell\right) / t$ which are equivalent to the eigenfunctions found for $\lambda_{0 T}=-1$.

We may also show that, for $\theta$-dependent perturbations (with $m=1$ ), the eigenvalue $\lambda_{1 S}=-\frac{1}{2}$ is associated with spatial translations. These transformations are defined by $r \longmapsto r+\delta \mathrm{e}^{\mathrm{i} \theta}$ and the image of the self-similar solution becomes

$$
\begin{aligned}
& u_{\delta}=\frac{1}{2} k t^{-1 / 2}\left\{U_{0}(\xi)+\delta \frac{\mathrm{e}^{\mathrm{i} \theta}}{k t^{1 / 2}}\right\}, \\
& h_{\delta}=\frac{1}{4} k^{2} t^{-1}\left\{H_{0}(\xi)+\delta \frac{\xi \mathrm{e}^{\mathrm{\theta} \theta}}{k t^{1 / 2}}\right\} .
\end{aligned}
$$

The coefficients of the infinitesimal transformation are thus given by $\left[\partial u_{\delta} / \partial \delta\right]_{\delta=0}=$ $\mathrm{e}^{\mathrm{i} \theta} /\left(k t^{1 / 2}\right)$ and $\left[\partial h_{\delta} / \partial \delta\right]_{\delta=0}=\xi \mathrm{e}^{\mathrm{i} \theta} /\left(k t^{1 / 2}\right)$.

With $m=1$ and $\lambda_{1 S}=-\frac{1}{2}$, the expressions from the linear stability analysis yield $\Phi_{1 S} \sim \xi \mathrm{e}^{\mathrm{i} \theta}$ and $\Psi_{1 S} \sim \mathrm{e}^{\mathrm{i} \theta}$ which are equivalent to the coefficients of the infinitesimal transformation.

These calculations show that imposing the eigenfunctions that correspond to eigenvalues $\lambda_{0 V}, \lambda_{0 T}$, and $\lambda_{1 S}$ on the similarity solution (2.7)-(2.10) causes no change in its structure, but merely redefine the spatial and temporal origins or the volume of fluid transported by the flow. The effects of these eigenfunctions and the loss of similarity can always be negated by appropriately redefining the variables $V, t$, and $r$.

\section{Concluding remarks}

We have studied the development of inertial gravity currents that arise as a result of compositional differences between the intruding and ambient fluids. By introducing series expansions about the self-similar solutions, we have carried out linear stability analyses to investigate the time evolution of small disturbances that are imposed on these solutions. In axisymmetric geometry, we have established that the self-similar solution is linearly stable to disturbances that involve both radial and azimuthal dependences by proving that all eigenvalues have negative real parts. We have derived an asymptotic formula for the eigenvalues which, in the absence of $\theta$-dependence, reduces to a formula that improves on the asymptotic expression obtained by GR. 
The asymptotic analysis indicates that, in the $\operatorname{limit}\left|\operatorname{Im}\left(\lambda_{m n}\right)\right| \rightarrow \infty$, for fixed values of the Froude number in the interval $0<F r \leqslant \sqrt{2}$, the value of $\operatorname{Re}\left(\lambda_{m n}\right)=\Lambda_{m n}$, that determines the rate of decay of the disturbance eigenfunctions, approaches a constant $\Lambda_{\infty}(F r)$ between $-\frac{1}{4}$ and 0 . For example, when $F r=1$, the asymptotic rate of decay is given by $t^{-0.22}$. Numerical calculations have shown that the direction from which the asymptotic value of $\Lambda_{m n}$ is approached is a function of dependence on $\theta$. For disturbances involving eigenfunctions with $m<2$, the approach is from above while, for $m \geqslant 2$, it is from below. This is important because it means that disturbances involving only $m \geqslant 2$, with a leading-order rate of decay given by $t^{\Lambda_{\infty}}$, decay faster than disturbances involving only $m<2$. Hence axisymmetric perturbations decay less rapidly than asymmetric ones in general.

We have also shown the relationship between the perturbation eigenfunctions and the symmetry transformations of the self-similar solution. The calculations demonstrate that three of the eigenfunctions correspond to a change of volume and changes of spatial and temporal origins. Thus, when considering to which similarity solution the flow evolves from certain initial conditions, by appropriate definition of volume carried by the current and spatial and temporal origin, these perturbations may be absorbed into the 'target' similarity solution. Additionally, for two-dimensional currents, we have proved analytically (in the Appendix) that all eigenvalues have a real part equal to $-\frac{1}{2}$ which indicates that the amplitude of all non-trivial eigenfunctions of the disturbances decay like $t^{-1 / 2}$.

These results are useful because they further assert the value of similarity solutions for modelling the spread of a finite volume of dense fluid through a less dense environment. We have demonstrated that the similarity solution is linearly stable to perturbations and thus we anticipate that it provides the intermediate asymptotics for this class of motion. Moreover, since axisymmetric perturbations generally decay slowest, we expect laboratory realizations of these flows to develop a radial symmetric profile.

\section{Appendix. Two-dimensional currents}

We study the two-dimensional compositional gravity current produced by the instantaneous release of a finite volume of a dense fluid within a less dense ambient. The dimensionless governing equations are given by

$$
\begin{aligned}
\partial_{t} h+\partial_{x}(u h) & =0, \\
\partial_{t} u+\partial_{x}\left(\frac{1}{2} u^{2}+h\right) & =0,
\end{aligned}
$$

where $x$ denotes the horizontal space coordinate, $u$ now represents the depth-averaged velocity in the $x$-direction, and lengths and times have been made dimensionless with respect to $h_{0}$ and $\left(h_{0} / g^{\prime}\right)^{1 / 2}$, respectively. The boundary conditions take the form

$$
\int_{0}^{x_{F}} h \mathrm{~d} x=V, \quad u(0, t)=0, \quad \dot{x}_{F}^{2}=F r^{2} h\left(x_{F}, t\right), \quad \dot{x}_{F}=u\left(x_{F}, t\right),
$$

where the dimensional volume per unit width $\tilde{V}=V h_{0}^{2}$.

Then, looking for a solution of the form

$$
u(x, t)=\frac{2}{3} \kappa t^{-1 / 3} U_{0}(y), \quad h(x, t)=\frac{4}{9} \kappa^{2} t^{-2 / 3} H_{0}(y), \quad y=\frac{x}{a\left(V t^{2}\right)^{1 / 3}},
$$


where $\kappa$ and $a=\kappa V^{-1 / 3}$ are constants, with the latter to be determined such that $y=1$ at the front of the current, yields (Fannelop \& Waldman 1972; Hoult 1972)

$$
\begin{aligned}
& U_{0}(y)=y, \\
& H_{0}(y)=\frac{1}{4}\left(y^{2}+\Pi\right),
\end{aligned}
$$

where $\Pi=4 / F r^{2}-1$ and $\kappa=\left[\left(27 V F r^{2}\right) /\left(12-2 F r^{2}\right)\right]^{1 / 3}$. The development of this similarity solution subsequent to the introduction of a small perturbation is studied by introducing the expansions

$$
\begin{aligned}
u(x, t) & =\frac{2}{3} \kappa t^{-1 / 3}\left\{U_{0}(y)+\delta U_{1}(y, t)+\cdots\right\}, \\
h(x, t) & =\frac{4}{9} \kappa^{2} t^{-2 / 3}\left\{H_{0}(y)+\delta H_{1}(y, t)+\cdots\right\}, \\
x_{F}(t) & =\kappa t^{2 / 3}\left\{1+\delta y_{F 1}(t)+\cdots\right\},
\end{aligned}
$$

where $\delta \ll 1$ is the amplitude of the perturbation. We substitute these expansions into the governing equations (A 1) and (A 2), and balance terms of equal powers of $\delta$. The terms at $O(1)$ yield the similarity solution. At $O(\delta)$, the equations of mass and momentum conservation become, respectively,

$$
\begin{aligned}
\frac{3}{2} t \partial_{t} H_{1}+\partial_{y}\left(H_{0} U_{1}\right) & =0, \\
\frac{3}{2} t \partial_{t} U_{1}+\partial_{y} H_{1}+\frac{1}{2} U_{1} & =0 .
\end{aligned}
$$

The boundary conditions are given by

$$
U_{1}(0, t)=0, \quad U_{1}(1, t)=\frac{3}{2} t \dot{y}_{F 1}, \quad H_{1}(1, t)=\frac{3}{4}(\Pi+1) t \dot{y}_{F 1}+\frac{1}{2} \Pi y_{F 1} .
$$

Eliminating $H_{1}$ between equations (A 7) and (A 8) yields a second-order partial differential equation in $U_{1}$

$$
9 t \partial_{t}\left(t \partial_{t} U_{1}\right)+3 t \partial_{t} U_{1}=4 \partial_{y} \partial_{y}\left(H_{0} U_{1}\right) .
$$

In addition to the first two conditions in (A 9), the solution to this equation must satisfy the following boundary condition:

$$
\partial_{y} U_{1}(1, t)=-\frac{3}{2}\left(5 t \dot{y}_{F 1}+3 t^{2} \ddot{y}_{F 1}\right),
$$

obtained by evaluating (A 7) at $y=1$.

We use the method of separation of variables and look for a solution of the form

$$
U_{1}(y, t)=\sum_{n=0}^{\infty} a_{n} t^{\lambda_{n}} \Psi_{n}(y), \quad y_{F 1}(t)=\sum_{n=0}^{\infty} b_{n} t^{\lambda_{n}},
$$

where $a_{n}$ and $b_{n}$ are constants, and $\lambda_{n}=\Lambda_{n}+\mathrm{i} \Omega_{n}$ are the eigenvalues of the system. The eigenfunctions $\Psi_{n}$ then satisfy the ordinary differential equation

$$
\left(y^{2}+\Pi\right) \Psi_{n}^{\prime \prime}+4 y \Psi_{n}^{\prime}+\left(1-3 \lambda_{n}\right)\left(2+3 \lambda_{n}\right) \Psi_{n}=0,
$$

subject to the boundary conditions

$$
\Psi_{n}(0)=0, \quad \Psi_{n}(1)=\frac{3}{2} \lambda_{n} b_{n} / a_{n}, \quad \Psi_{n}^{\prime}(1)=-\frac{9}{2} \lambda_{n}\left(\lambda_{n}+\frac{2}{3}\right) b_{n} / a_{n} .
$$

The substitution $y^{2}=-\Pi \xi$ transforms equation (A 13) to

$$
\xi(1-\xi) \Psi_{n}^{\prime \prime}+\left(\frac{1}{2}-\frac{5}{2} \xi\right) \Psi_{n}^{\prime}-\frac{1}{4}\left(1-3 \lambda_{n}\right)\left(2+3 \lambda_{n}\right) \Psi_{n}=0,
$$


which is the hypergeometric differential equation (e.g. Abramowitz \& Stegun 1965). The solution of (A 15) which satisfies the $(\mathrm{A} 14 a, b)$ is given by

$$
\Psi_{n}=\frac{3}{2} \lambda_{n} b_{n} y \frac{{ }_{2} F_{1}\left(1-\frac{3}{2} \lambda_{n}, \frac{3}{2}+\frac{3}{2} \lambda_{n} ; \frac{3}{2} ;-y^{2} / \Pi\right)}{a_{n 2} F_{1}\left(1-\frac{3}{2} \lambda_{n}, \frac{3}{2}+\frac{3}{2} \lambda_{n} ; \frac{3}{2} ;-1 / \Pi\right)} .
$$

This solution does not satisfy the third boundary condition, (A 14c) in general. Requiring the solution to also satisfy this condition gives an equation that specifies the eigenvalues of the system. Substituting (A 16) into (A 14c) yields

$$
\frac{{ }_{2} F_{1}\left(2-\frac{3}{2} \lambda_{n}, \frac{5}{2}+\frac{3}{2} \lambda_{n} ; \frac{5}{2} ;-1 / \Pi\right)}{{ }_{2} F_{1}\left(1-\frac{3}{2} \lambda_{n}, \frac{3}{2}+\frac{3}{2} \lambda_{n} ; \frac{3}{2} ;-1 / \Pi\right)}=\frac{3 \Pi}{2-3 \lambda_{n}}
$$

which must be satisfied by all the eigenvalues $\lambda_{n}$. We note that boundary condition (A $14 c$ ) is trivially satisfied by (A 16) for the eigenvalues $\lambda_{V}=0$ and $\lambda_{T}=-1$. These two eigenvalues are associated with change of mass and time-shift symmetry transformations of the problem, respectively.

GR have proved that $\Lambda_{n}<0$ for this problem. We now prove analytically that $\Lambda_{n}=-\frac{1}{2}$ for all $n$. Using the formula (taken from Abramowitz \& Stegun 1965)

$$
{ }_{2} F_{1}(a, b ; c ; z)=(1-z)^{c-a-b}{ }_{2} F_{1}(c-a, c-b ; c ; z),
$$

equation (A 17) can be transformed into

$$
\frac{{ }_{2} F_{1}\left(\frac{1}{2}+\frac{3}{2} \lambda_{n},-\frac{3}{2} \lambda_{n} ; \frac{5}{2} ;-1 / \Pi\right)}{{ }_{2} F_{1}\left(\frac{1}{2}+\frac{3}{2} \lambda_{n},-\frac{3}{2} \lambda_{n} ; \frac{3}{2} ;-1 / \Pi\right)}=\frac{3(1+\Pi)}{2-3 \lambda_{n}} .
$$

Then applying the contiguous relation (Abramowitz \& Stegun 1965)

$$
z(c-b){ }_{2} F_{1}(a, b ; c+1 ; z)=c_{2} F_{1}(a-1, b ; c ; z)-c(1-z)_{2} F_{1}(a, b ; c ; z)
$$

gives

$$
{ }_{2} F_{1}\left(-\frac{3}{2} \lambda_{n}-1, \frac{1}{2}+\frac{3}{2} \lambda_{n} ; \frac{3}{2} ;-1 / \Pi\right)=0 .
$$

This equation implies that $F$ equals its complex conjugate $\bar{F}$, where $F$ denotes the hypergeometric function in (A 21). This is only possible if the real parts of the first two arguments of $F$ are equal, that is,

$$
-\frac{3}{2} \Lambda_{n}-1=\frac{1}{2}+\frac{3}{2} \Lambda_{n}
$$

Thus we deduce the result $\Lambda_{n}=-\frac{1}{2}$ which not only establishes that the self-similar solution (A 4)-(A 6) is linearly stable but also implies that the perturbation eigenfunctions all decay at the same rate of $t^{-1 / 2}$. The latter is a remarkable result which represents a departure from the usual situation where different eigenfunctions decay at different rates.

\section{REFERENCES}

Abramowitz, M. \& Stegun, I. A. 1965 Handbook of Mathematical Functions. Dover. Benjamin, T. B. 1968 Gravity currents and related phenomena. J. Fluid Mech. 31, 209-248.

Bonnecaze, R. T., Hallworth, M. A., Huppert, H. E. \& Lister, J. R. 1995 Axisymmetric particledriven gravity currents. J. Fluid Mech. 294, 93-121.

Fannelop, T. K. \& Waldman, G. D. 1972 Dynamics of oil slicks. AIAA J. 10, 506-510.

FAY, J. A. 1969 The spread of oil slicks on a calm sea. In Oil on the Sea (ed. D. P. Hoult), pp. 43-63. Plenum. 
Grundy, R. E. \& RotTman, J. W. 1985 The approach to self-similarity of the solutions of the shallow-water equations representing gravity-current releases. J. Fluid Mech. 156, 39-53.

Hallworth, M. A., Hogg, A. J. \& Huppert, H. E. 1998 Effects of external flow on compositional and particle gravity currents. J. Fluid Mech. 359, 109-142.

Hallworth, M. A., Huppert, H. E., Phillips, J. C. \& Sparks, R. S. J. 1996 Entrainment into two-dimensional and axisymmetric turbulent gravity currents. J. Fluid Mech. 308, 289-311.

Harris, T. C., HogG, A. J. \& Huppert, H. E. 2001 A mathematical framework for the analysis of particle-driven gravity currents. Proc. R. Soc. Lond. A 457, 1241-1272.

Hogg, A., Ungarish, M. \& HupPert, H. 2000 Particle-driven gravity currents: asymptotic and box model solutions. Eur. J. Mech. B/Fluids 19, 139-165.

Hoult, D. P. 1972 Oil spreading on the sea. Annu. Rev. Fluid Mech. 4, 341-368.

HupPert, H. E. \& Simpson, J. E. 1980 The slumping of gravity currents. J. Fluid Mech. 99, 785-799.

vON KÁRmán, T. 1940 The engineer grapples with non-linear problems. Bull. Am. Math. Soc. 46, 615-683.

Mathunjwa, J. S. 2004 Self-similarity in inertial and porous media gravity currents. PhD Thesis, University of Bristol.

Mathunjwa, J. S. \& HogG, A. J. 2006 Self-similar gravity currents in porous media: Linear stability of the Barenblatt-Pattle solution revisited. Eur. J. Mech. B/Fluids 25, 360-378.

RotTMAn, J. W. \& Simpson, J. E. 1983 Gravity currents produced by instantaneous releases of heavy fluid in a rectangular channel. J. Fluid Mech. 135, 95-100.

Shin, J. O., Dalziel, S. B. \& Linden, P .F. 2004 Gravity currents produced by lock exchange. J. Fluid Mech. 521, 1-34.

Simpson, J. E. 1997 Gravity Currents in the Environment and the Laboratory. Cambridge University Press. 